New Comer Corner

Johanna M. Askeridis*

\title{
Studienaufenthalt in Südkorea: Zwischen Kursen, Karaoke und Corona
}

https://doi.org/10.1515/iwp-2020-2104

Die Luft ist warm und feucht und das Zirpen der Zikaden frech, als ich aus dem Bus steige, zwei Koffer und einen Rucksack als Gepäck. Vor mir erhebt sich das zwölfstöckige Studentinnen-Gebäude des Yangseongjae-Wohnheims, in dem ich für die nächsten zwei Semester wohnen soll. Es ist der 30. August 2019 und ich befinde mich auf dem Campus der Chungbuk National University (CBNU) in Cheongju, Südkorea. Langsam wird mir bewusst, dass es die Realität ist: Die Hälfte meines Joint-Degree-Masters Global Studies on Management and Information Science (GLOMIS) werde ich hier verbringen. $\mathrm{Zu}$ diesem Zeitpunkt ahne ich noch nicht, dass ich nach einem Semester wieder nach Hause fliegen werde. Das neuartige Coronavirus SARSCoV-2 wird meinen Plänen einen Strich durch die Rechnung machen. Bis dahin liegen aber noch sechs spannende Monate vor mir, in denen ich einen anderen Studienalltag und eine fremde Kultur aus nächster Nähe kennenlernen darf - einschließlich wie diese mit dem Ausbruch des Coronavirus umgeht.

Mein Name ist Johanna Askeridis und ich bin zum Zeitpunkt meiner Reise nach Korea eine 22-jährige Masterstudentin von der Universität Hildesheim. Ich studiere im vom Deutschen Akademischen Austauschdienst (DAAD) geförderten Joint-Degree-Programm GLOMIS der deutschen Universität Hildesheim und österreichischen KarlFranzens-Universität Graz in Kooperation mit den südkoreanischen Universitäten Chungbuk National University in Cheongju sowie Pai Chai University in Daejeon. Studierende in diesem Programm verbringen zwei Semester an ihrer Heimatuniversität und zwei Semester an einer der Partneruniversitäten und erhalten einen gemeinsamen Masterabschluss beider Universitäten.

Am 2. September beginnt die Vorlesungszeit. Bis sie am 20. Dezember wieder endet, belege ich drei Kurse für je 10 Credits: Management Information Systems, Special

*Kontaktperson: Johanna M. Askeridis, Universität Hildesheim, Institut für Informationswissenschaft und Sprachtechnologie, Universitätsplatz 1, 31141 Hildesheim,

E-Mail: askeridi@uni-hildesheim.de
Topics on Data Mining und ein Kolloquium, welches auf die Masterarbeit vorbereitet. Neben mir sind dieses Semester sechs weitere Studierende im GLOMIS-Programm an der CBNU, mit denen ich den Stundenplan teile. Ich spreche ein wenig Koreanisch, doch der Unterricht findet auf Englisch statt. Für Studierende wie mich, die noch keine Vorkenntnisse aus dem Managementbereich mitbringen, ist der Perspektivwechsel zu Management Information Systems besonders lehrreich. Mithilfe des Lehrbuchs „Information Systems for Managers: With Cases“ (Piccoli \& Pigni, 2016) gibt die gleichnamige Lehrveranstaltung einen strukturierten Überblick über das Feld. In die meisten Lehrveranstaltungen ist wirtschaftliches Denken integriert. Aber auch nicht-fachlich unterscheidet sich das Studium in Südkorea von dem in Deutschland. Beispielsweise fällt mir auf, dass die Trennung zwischen Arbeit und Privatleben in Südkorea nicht so streng ist. So ist es nicht unüblich, dass man als Masterstudent auch mal von seinem Professor zum Essen eingeladen wird. Und tatsächlich stimmt, dass die südkoreanischen Studierenden viel Fleiß an den Tag legen. $\mathrm{Zu}$ studieren, vor allem an einer renommierten Universität, hat hier einen sehr hohen Stellenwert, ähnlich eines Etappenziels im Leben. Meine koreanischen Kommilitonen sind entsprechend viel mit dem Studium beschäftigt, und auch ich habe über die drei Kurse hinweg jede Woche eine Teilprüfungsleistung zu erbringen - eine Präsentation, eine Abgabe, eine schriftliche Prüfung. Irgendwas steht immer an. Trotzdem finde ich irgendwie noch Zeit, Cheongju und Südkorea zu erkunden, auszugehen, Leute kennenzulernen, Dinge zu unternehmen.

Wie sieht der Alltag eines ausländischen Masterstudierenden in Südkorea aus? Ich teile mir ein kleines Zimmer mit einer Austauschstudentin aus Berlin im Wohnheim auf dem Campus. Auf den ersten Blick wirkt es nicht gerade luxuriös, man gewöhnt sich jedoch schneller daran, als man denkt und bald wird das kleine Wohnheimzimmer im dritten Stock zum Zuhause. Eine Küche gibt es nicht, stattdessen bekommen wir drei Mal am Tag eine Mahlzeit in der Wohnheimkantine. Wenn ich frühstücken möchte, stehe ich um 8.30 Uhr auf. Bis zum Mittagessen um 12 Uhr arbeite ich an meinen diversen Kursprojekten, 




Abbildung 1: Das Studentinnen-Gebäude des YangseongjaeWohnheims an der Chungbuk National University, mein Zuhause für die Zeit in Südkorea.

danach habe ich entweder Unterricht oder unternehme etwas mit Freunden. Oft geht es dabei nach koreanischer Manier in eines der diversen Study-Cafés rund um den Campus. Mit einem Kaffee oder Tee bewaffnet wird der Laptop aufgeklappt und die Arbeit fortgesetzt. Manchmal gehen wir auch einfach aus, um zu quatschen, meistens findet sich im Café Zeit für beides. Um 18 Uhr gibt es Abendessen. Die vielen, günstigen Restaurants am Campus laden uns oft dazu ein, auch auswärts essen zu gehen, denn wer nach Südkorea reist, sollte unbedingt die lokale Küche genießen! Viele Abende verbringen wir ausgelassen im Noraebang, einem kleinen Karaoke-Raum mit zwei Mikrophonen - 25 Minuten für 1000 Won, etwa 75 Cent. Fleiß am Tag und Ausgelassenheit bei Nacht gehören zum koreanischen Studienalltag dazu.

Zeit zum Reisen bleibt auch. Südkorea ist ein kleines, bergiges Land. Reisen per Bus ist hier normal und komfortabel. Für eine anderthalbstündige Direktfahrt von Cheongju nach Seoul zahle ich umgerechnet zwischen 6,50 Euro und 8,50 Euro, je nachdem wie bequem der Bus ist (und der 8,50-Euro-Bus ist wirklich sehr bequem!). Ich besuche die Millionenmetropole Seoul, Gangneung an der
Ostküste und das nahgelegene Daejeon, in dem die zweite koreanische GLOMIS-Universität liegt. Die großen Städte im Süden wie Busan, traditionellere Orte wie Jeonju oder die Urlaubsinsel Jeju wollte ich mir eigentlich im Frühling noch ansehen.

Von Mitte Dezember bis Anfang März ist vorlesungsfreie Zeit. Es wird ruhig im Wohnheim, fast alle Studierenden ziehen über die Ferien aus. Von allen GLOMIS-Studierenden bin ich die Einzige, die in den Semesterferien nicht nach Hause fliegen möchte. Es liegen also zweieinhalb ruhige Monate vor mir. Weihnachten, was in Südkorea eher ein Feiertag für Pärchen ist, verbringe ich mit einer Freundin aus Deutschland in Seoul. Zu Neujahr werde ich von einer koreanischen Freundin zu ihrer Familie nach Hause eingeladen. Das heißt: Gemeinsam aufs neue Jahr anstoßen, um 2 Uhr ins Bett gehen und um 6 Uhr wieder aufstehen, um auf einen Berg zu wandern und den ersten Sonnenaufgang des Jahres zu sehen. Zwischen Ende Januar und Anfang Februar feiert Südkorea jedoch noch ein zweites Mal Neujahr, das traditionelle Mondneujahr Seollal. Es hat eine ähnlich große Bedeutung für die Familie wie Weihnachten für die Deutschen. Entsprechend unvergesslich ist es, dass eine weitere koreanische Freundin mich einlädt, zu ihrer Familie an die Südküste zu reisen und mit ihr Seollal zu feiern. Ich darf sogar bei den Ahnenritualen nicht bloß zuschauen, sondern selbst teilnehmen. So tief bin ich noch nie in die koreanische Kultur eingetaucht.

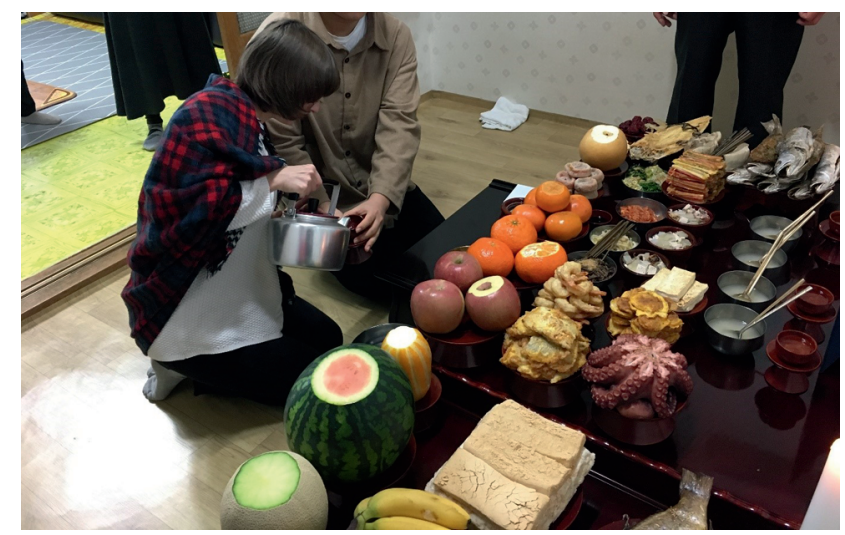

Abbildung 2: Beim traditionellen Mondneujahr Seollal darf ich an den Ahnenritualen teilnehmen.

Im Jahr 2020 fällt Seollal auf den 25. Januar. Fünf Tage zuvor, am 20. Januar, wurde der erste COVID-19-Fall in Südkorea bestätigt. Ich ahne noch nicht, dass ich keinen Monat später nach Hause fliegen werde. Zunächst habe ich das Gefühl, Südkorea habe die Situation im Griff. Die Zahl der Neuinfektionen bleibt gering, die Infektionswege sind klar nachvollziehbar. Ausgehen soll man nicht, 
selbst wenn auf Ausgangs- oder Kontaktsperren bis zum Ende meiner Zeit dort verzichtet wird. Die Südkoreaner sind diszipliniert und halten sich an die staatlichen Empfehlungen. In diesem Zuge kommt das Sozialleben zum Stillstand.



Abbildung 3: Ein Informationsplakat zu Verhaltenshinweisen wegen der Ausbreitung des Coronavirus in einer U-Bahn-Station in Seoul, extra für Ausländer auf Englisch und Chinesisch.

Mit den steigenden Infektionszahlen sehe ich Veränderungen: Überall tauchen Informationsplakate auf mit Hinweisen zum Verhalten im Angesicht der Ausbreitung des Coronavirus. Desinfektionsmittel stehen auf kleinen runden Tischchen in öffentlichen Gebäuden oder Läden. Beinahe jeder trägt einen Mund-Nase-Schutz - ich natürlich auch. In öffentlichen Verkehrsmitteln gibt es Durchsagen, man möge bitte eine Maske tragen. Vor dem Betreten einiger Gebäuden, wie dem International Office an der CBNU oder der Wohnheimkantine, müssen die Hände desinfiziert werden und es herrscht eine Maskenpflicht.

Mitte Februar beschließt das Bildungsministerium, den Semesterbeginn um zwei Wochen zu verschieben, auf Mitte März. In der ersten Zeit soll der Unterricht digi- tal stattfinden. Alle Studierenden, die zum Semesterstart über China einreisen, müssen zunächst zwei Wochen in Quarantäne. Schließlich höre ich, mein Wohnheim werde geschlossen, falls es dort einen COVID-19-Fall geben sollte.

Das meiste dieser Informationen wird nur auf Koreanisch veröffentlicht. Ich bin in der Regel darauf angewiesen, dass mich andere über wichtige Punkte informieren oder, dass das Übersetzungsprogramm gut funktioniert, wenn ich mehr Details verstehen möchte, als meine Koreanisch-Grundkenntnisse hergeben. In dieser Zeit kann niemand absehen, wie sich die Lage in Zukunft entwickeln wird. Viel über SARS-CoV-2 ist noch nicht bekannt. Ständig passiert etwas Neues, das mich mehr verunsichert als zuvor.

Letztlich beschließe ich, dass ich nach Deutschland zurückkehren möchte. Noch gibt es keine Fälle in Cheongju. Einen Tag, bevor ich zurückreise, höre ich von einer koreanischen Freundin, dass es einen explosionsartigen Anstieg der Fallzahlen in der Großstadt Daegu gegeben hat. Die Infektionswege sind plötzlich nicht mehr nachvollziehbar. Am 22. Februar komme ich zu Hause an. Jetzt gibt es auch Fälle in Cheongju. Daegu wird zum Risikogebiet erklärt werden. Gerade rechtzeitig, denke ich. Und endlich wieder gutes Brot.

Ich habe Südkorea mit einem lachenden und einem weinenden Auge verlassen. Abschiednehmen ist immer schwer, gerade wenn es übereilt ist. Ich hätte gerne all meine Pläne verwirklicht und mehr außergewöhnliche Dinge mit meinen koreanischen Freunden erlebt. Doch ich bin auch froh, während der Pandemie zu Hause zu sein, wo mir Konventionen bewusst sind, ich die Sprache fließend spreche, mich einschränkungslos informieren kann, und sozial aufgefangen werde. Die Zeit in Südkorea war lehrreich und spannend. Ich kenne nun auch mich selbst ein Stück besser als zuvor. Zwar war nicht alles schön und einfach - es gab Schwierigkeiten, Stress und interkulturelle Probleme - doch auch das gehört dazu. Daran wächst man, schaut zurück und alles in allem ist man dann doch ziemlich stolz auf sich.

\section{Fünf Hinweise für Studierende, die ein Semester in Südkorea verbringen möchten:}

Lernt vor der Anreise die Sprache! Englisch wird in Südkorea auch von der jüngeren Generation kaum gesprochen. Zumindest die Schriftzeichen, die sehr einfach zu lernen sind, solltet ihr lesen können. 
Interkulturelle Missverständnisse sind nicht $\mathrm{zu}$ vermeiden! Die südkoreanische Kultur unterscheidet sich sehr von der unseren. Euch wird nicht alles an ihr gefallen. Den Koreanern wird nicht alles an euch gefallen. Das ist normal und Teil des interkulturellen Lernprozesses. Bleibt offen für neue Sichtweisen und lasst euch nicht entmutigen!

Die koreanische Gesellschaft ist sehr homogen! Es ist doch irgendwie ungewöhnlich, einen nicht-asiatisch anmutenden Menschen auf der Straße zu sehen, vor allem außerhalb Seouls (und manchmal auch da). Die Koreaner haben unter Umständen Respekt davor, dass ihr anders ausseht oder eine andere Sprache sprecht. Seid euch dessen bewusst.

Stellt euch auf eine Fleisch lastige Küche ein! Vegane oder vegetarische Ernährung ist in Südkorea noch nicht in der Mitte der Gesellschaft angekommen. Die meisten Gerichte enthalten Fisch oder Fleisch zumindest in geringen Mengen. Wenn man sagt „ohne Fleisch“ bekommt man ggf. Würstchen oder Schinken - Das ist ja kein „Fleisch“ in dem Sinne.

Lasst euch auf Land und Leute ein! Seid neugierig, probiert aus, stellt Fragen! Ihr seid hier Gast, Ihr seid hier, um zu lernen. Probiert das Essen, sprecht die Sprache, geht Karaoke singen. Versucht unter Koreaner zu kommen, auch wenn es bequemer ist unter Ausländern $\mathrm{zu}$ bleiben. Es ist eine einmalige Gelegenheit.

\section{Literatur}

Piccoli, G., \& Pigni, F. (2016). Information systems for managers: With cases (Ausgabe 3.1). Burlington: Prospect Press.

Deskriptoren: Hochschulausbildung, Südkorea, Kultur



Johanna M. Askeridis

Universität Hildesheim

Institut für Informationswissenschaft und

Sprachtechnologie

Universitätsplatz 1

31141 Hildesheim

askeridi@uni-hildesheim.de 